\title{
Comparative evaluation of the immune responses of seven chicken ecotypes to vaccination against New- castle disease
}

\author{
Edilu Jorga Sarba ${ }^{1 \uparrow}$, Endashaw Kemal ${ }^{2}$,Eyob Galan ${ }^{3}$, Teshale Sori ${ }^{2}$, Yilkal Asfaw \\ Woube, ${ }^{4}$ and Reta Duguma Abdi $i^{5 \mp a}$ \\ ${ }^{1}$ College of Agriculture and Veterinary Science, Ambo University, P.O.Box 19, Ambo, Ethiopia \\ ${ }^{2}$ College of Veterinary Medicine and Agriculture, Addis Ababa University, P.O.Box 34, Bishoftu, \\ Ethiopia \\ ${ }^{3}$ School of Veterinary Medicine, Walaita Sodo University P.O.Box 138, Sodo, Ethiopia \\ ${ }^{4}$ Center of Excellence for Food Animal Health and Food Safety, College of Veterinary Medicine, \\ Tuskegee University, Tuskegee, AL 36088, USA \\ ${ }^{5}$ Department of Biomedical Sciences, College of Veterinary Medicine, Long Island University, \\ Greenvale, New York, USA \\ " Equal contribution \\ ${ }^{a}$ Corresponding author: reta.abdi@liu.edu
}

\begin{abstract}
Newcastle disease (ND) is a highly contagious viral disease of poultry with high mortality. The local velogenic viral pool of the ND strains influences its severity and occurrence. ND vaccination is the most feasible approach to control the disease. However, some ND-vaccinated groups within chicken populations are susceptible to velogenic ND infection developing outbreaks with marked pathological lesions and shedding of the virus. Vaccine strain-related factors as well as inadequate vaccine application and delivery methods during vaccination might explain the suboptimum ND vaccine efficacy. In this study, however, we propose that host factors may contribute to the suboptimal vaccine efficacy in vaccinated chickens. We, therefore, compared the immune response of five Ethiopian chicken ecotypes to ND immunization in the presence of two reference breeds (Fayoumi and Bovans). All chickens received initial immunization at age of 21 days with HB1 ND vaccine followed by two-times LaSota booster immunization at age 50 and 120 days. Subsequently, serum was collected fortnightly post-vaccination at age 35,65 , and 135 days for immune response analysis using the hemagglutination inhibition (HI) test. HI antibody was significantly higher at days $135>65>35$ in each ecotype following the third, second, and first vaccination, respectively. The different chicken
\end{abstract}


ecotypes had significant differences in HI antibody response to the ND vaccination. Accordingly, the HI titer was significantly higher in Jarso $>$ Cheffe $>$ Fayoumi $>$ Arsi $>$ Bovans $>$ Tepi $>$ Horro suggesting antibody titer and ND vaccine efficacy of the ND vaccine depends on host factors. Moreover, some chicken groups within each ecotype had low HI titer. Chicken ecotypes with weak immune responses may not completely clear the virus from their body; thus, they can serve as a reservoir host by maintaining the ND virus. We conclude that herd immunity level and blanket vaccination program based on the results of a single host genetic group can be misleading during developing and recommending a new vaccine. Hence, understanding the host determinant factors in the immune response during vaccination can lead to improved efficacy and protection against ND in chicken populations.

Keywords: Chicken; Ecotypes; Immune Response; Newcastle Disease; Vaccination, $H I$.

\section{Introduction}

The chicken industry in Ethiopia is dominated by local chicken ecotypes with low input - low output production systems in the villages (Duguma, 2009). Local chicken ecotypes contribute about $97 \%$ to the sector whereas the modern commercial production system, however, is very low (2-3\%) (Duguma, 2009; Gulilat et al., 2021). The Ethiopian local chicken ecotypes are a pool of highly heterogeneous genetic resources (Hassen et al. 2009; Goraga et al., 2012). They demonstrate heterogeneity in production performance (Duguma 2009), morphology and phenotypes (Duguma, 2006; Dana et al., 2010a; Melesse and Negesse, 2011), and individual and ecotype variation in carrying diverse polypathogens as well as parasite loads (Ashenafi et al., 2004; Duguma, 2009; Luu et al., 2013; Bettridge et al., 2014; Sarba et al., 2019). The heterogeneity of local chickens is a dynamic process attributable to continuous selection pressure imposed on them by two broad forces (Duguma, 2009). These include (1) selection by natural phenomena including diseases, starvation, predators, and ecological stress factors; and (2) selection by production and marketing processes including preferred traits for the market, reproduction (stocking), and slaughter or gift for socio-cultural needs (Duguma, 2009; Dana et al., 2010b). Pathogens causing diseases can impose selective pressure on their hosts by inducing host genetic diversity subsequently grouping the host population into a continuum, ranging from susceptible to resistant groups (Hamilton, 1982; Lars et al., 2009; Grubaugh et al., 2015). A marked continuum among Ethio- 
pian chicken ecotypes had been reported in Marek's disease virus infection and in their responses to immunization, ranging from highly susceptible to highly resistant ecotypes (Duguma et al., 2005; Duguma et al., 2006).

One of the major pathogenic diseases of chicken in Ethiopia is ND, which is circulating in all regions causing high morbidity and mortality at particular seasons (Chaka et al., 2012, 2013a, b; de Almeida et al., 2013; Fentie et al., 2014a,b; Mulisa et al., 2014; Bettridge et al., 2014). ND outbreaks vary geospatially in village backyard chicken production systems (Miguel et al., 2013; Snoeck et al., 2013) because of the composition of the local viral genotype population diversity (Alexander, 2011; Snoeck et al., 2013; Fentie et al., 2014b; Mulisa et al., 2014). Globally, the ND virus is classified into two classes based on their genome sequence similarity, namely class I and II whereas further phylogenetic analysis of the genome sequence of viral isolates shows class I has a single genotype and class II has 18 genotypes (Dimitrov et al., 2016). Other researchers have previously reported that 16 different ND genotypes are present globally, being distributed distinctly on the different landscapes, resulting in variation in ND outbreaks based on geographic location (Diel et al., 2012; Snoeck et al., 2013). Such spatial variation in local ND strains can contribute to vaccine mismatch (Degefa et al., 2004; van Boven et al., 2008; Dortmans et al., 2012), which may impair the effectiveness of ND control program due to low vaccine efficacy in the vaccinated-chickens (partial immunity and viral shedding) (van Boven et al., 2008; Dortmans et al., 2012; Fentie et al., 2014a). Contrarily, a study suggests that the ND vaccine may be effective (i.e. no mismatch in ND vaccine) but the vaccine failure could be due to inappropriate vaccination practice in the field (Dortmans et al., 2012). In addition to viral-specific factors, the heterogeneity of the ND viral population and the evolution of new ND genotypes in a particular area are consistently shaped by bird species-dependent factors through fitness competition among viral genotypes as well as a selective pressure imposed by bird species (Grubaugh et al., 2015). Thus, host genetics is an ecological condition that determines the geospatial variation in the local viral strain pool, ND lineage distributions, and ND outbreaks (Koenen et al., 2002; Grubaugh et al., 2015). Conversely, the ND virus creates host genetic diversity in their host population (Hamilton, 1982). Host-viral interaction and/or host-specific factors modify the immune responses and ND outbreaks (Grubaugh et al., 2015; Alfonso, 2021), affecting the ND control program of the disease. Disease occurrence and transmission dynamics are affected by variation in genetic diversity within a host species (Ostfeld and Keesing, 2012). Host genetics have a role in the modulation, mo- 
bilization, and operation of the host defense system in multiple ways on varying scales against deadly infectious pathogens to ensure health. Overall, host animals shape the ND viruses by creating genotype diversity (i.e., evolution of new ND virus through mutation) to adapt to the diverse host species in which new genotype can spillover from wild birds to domestic poultry (Alfonso, 2021). However, immunogenetic study on genotypes migrating between different interfaces is scarce. Little is known if such new genotype evolution causes antigenic variation (vaccine mismatch) in ND. Further study is required from both viral factors such as new genotype evolution and host factors on ND vaccine efficacy. The role of chicken genetic polymorphism on ND vaccine immune response is poorly understood in Ethiopia. This study, thus, has evaluated the comparative immune responses of seven chicken ecotypes to ND vaccine vaccination.

\section{Materials and methods}

\section{Study area}

The study was conducted on-station in the College of Veterinary Medicine and Agriculture of the Addis Ababa University (CVMA-AAU) at Bishoftu, Ethiopia. CVMA-AAU is located $47 \mathrm{~km}$ southeast of Addis Ababa, the capital city, having an altitude of 1850 meters above sea level and receiving an average rainfall of $866 \mathrm{~mm}$ per year. The maximum and minimum average temperatures are $26^{\circ} \mathrm{C}$ and $14^{\circ} \mathrm{C}$, respectively. The relative humidity of the study site ranges from 58.6 to $61.3 \%$ (Gari et al., 2018).

\section{Study animals}

Five indigenous chicken ecotypes from distinct locations of Ethiopia were selected for the study. The ecotypes are Arsi, Chefe, Jarso, Horro, and Tepi; located in the west, southwest, southeast, central, and east of the country, respectively (Figure 1). Bovans and Fayoumi breeds were included in the study as reference groups. Bovans is a commercial breed developed by Institut de Sélection Animale (ISA). Bovans breed is improved (known) for its high egg production. Fayoum chickens are known for their ability to tolerate hot weather, adapt easily to different climatic conditions, resistant to bacterial and viral diseases (Breed Profile: Egyptian Fayoumi Chicken - Backyard Poultry (iamcountryside.com)). Eggs of each ecotype were purchased from their respective local markets and transported to CVMA-AAU premises, where the study was conducted. At least two persons including DVM and MSc students were as- 
signed to an ecotype to travel and buy eggs from different Ethiopian locations. The travel schedule of all of them was in such a way that they return at the same time to incubate eggs of all ecotypes at a time. We purchased, transported, incubated, and hatched the eggs at the same time. As to the study chicken population, population genetic parameters of the chicken ecotypes (i.e. phenotypic characteristics and genetic distance and heterozygosity) have been previously measured revealing Ethiopian chicken ecotypes are genetically distinct from exotic commercial chickens and each other (Dessie, 2003; Hassen, 2007; Hassen et al., 2009; Dana, 2011; Goraga et al., 2012). Fayoumi eggs were obtained from Debre Zeit Agricultural Research Center while Bovans were obtained from Genesis Poultry Farm, franchised private farm with worldwide locations. The eggs were fumigated (2:1 ratio of formalin to potassium permanganate), incubated, and chickens hatched. Then, day-old chicks were transferred to experimental poultry houses of CVMA-AAU from hatcheries.

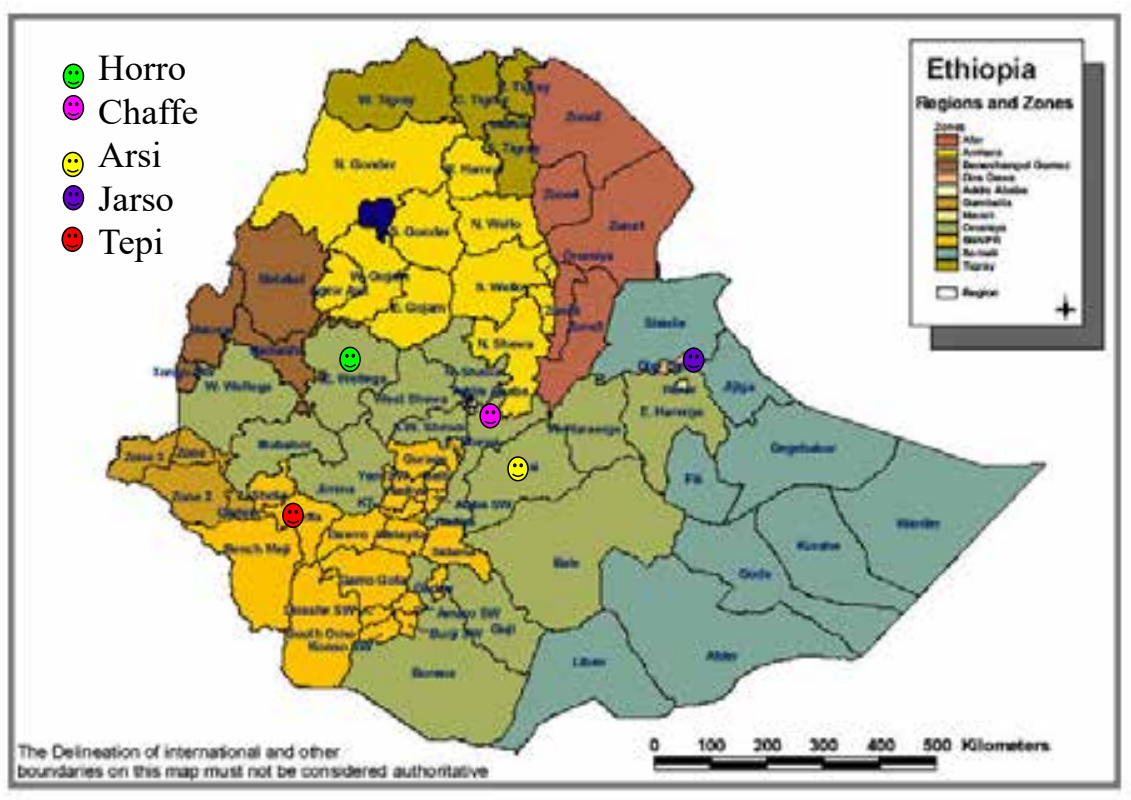

Figure 1. Map of Ethiopia showing the geographical origin of the selected chicken ecotypes in this study. 


\section{Preparation of animal house}

A $25 \mathrm{~m}$ by $8 \mathrm{~m}$ sized house was constructed on station and equipped with infrared-light bulbs. The ceiling, walls, and floor were disinfected using $1 \%$ formalin. Cleaned and disinfected local straw (Eragrostis teff) was spread over the floor. Drinkers, feeders, and buckets were cleaned and disinfected before the equipment was introduced into the houses.

\section{Study design, study animals' keeping, and vaccination}

All day-old chicks were vaccinated against Marek's disease. It was observed that ND HI titer from maternal immunity declines after 14 days in chicks (Abdi et al., 2016). HB1 vaccine was administered at 21 days of age whereas two doses of LaSota vaccine were given at days 50 and 120 . Both vaccines are produced at the National Veterinary Institute, Bishoftu, Ethiopia. A total of 260 chickens grouped into seven ecotypes were used in this study: 70 Cheffe, 40 Jarso, 21 Horro, 9 Arsi, 30 Tepi, 20 Bevans, and 70 Fayoumi chickens. The experimental design used in this study was a completely randomized design.

Chickens were kept in deep dry formalin disinfected beddings with teff-straw in their pens. Chicks were raised on an electrically heated brooder with infrared bulbs for light source according to Bovans breed management guide. Artificial lighting was provided for 24 hours starting from the first day until 20 weeks of age decreasing light in equally spaced intensity. The chickens were fed on starter commercial layer ration up to 8 weeks of age, grower commercial ration (9-20 weeks), and layer ration (21 weeks onwards) with standard formulations purchased from Genesis feed processing plant. Feeds and clean potable water were supplied to all stages of birds ad libitum.

\section{Serum sampling and antibody titer measurement}

Blood was collected from the wing vein at age 35, 65, and 135 days following vaccination on 21,50 , and 120 days of age to measure ND total serum haemagglutination inhibition (HI) as described previously (Abdi et al., 2016). The HI antibody titer of $\log _{2} \geq 3$ has been used to determine vaccination efficacy. It was reported previously that HI antibody titer of $\log _{2} \geq 3$ following vaccination correlated with effective protection to velogenic viral challenge (van Boven et al., 2008; Abdi et al., 2016). 


\section{Data analysis}

We used descriptive statistics (proportions) to summarize the HI antibody data using chicken breed as potential explanatory factors for the induced HI titer. The geometric mean value and standard deviation (SD) of HI antibody titers were determined and classified according to chicken ecotypes and a vaccine booster dose (single, second, and third dose). Post-vaccination mean HI antibody titers were analyzed using the General Linear Model of SPSS version 20 (IBM SPSS Statistics for Windows, Version 20.0. Armonk, NY: IBM Corp). Where HI results were significant, the least-square difference (LSD) was used to compare HI response by pair-wise breed comparisons. The proportion of chickens with HI antibody titer $\geq \log _{2}{ }^{3}$ between breeds was used as a cut-off value to decide the protective level (van Boven et al., 2008; Abdi et al., 2016). Significant differences were tested setting alpha at a $5 \%$ significance level.

\section{Results}

\section{Immune responses (HI titer) to ND vaccination within and among chicken ecotypes}

The immune response (HI titer) of chick ecotypes during ND immunization was heterogeneous; the response varied within and between chicken ecotypes as well as between each of the three-round ND vaccine booster doses (Table 1). The response of individual chickens during $1^{\text {st }}, 2^{\text {nd, }}$ and $3^{\text {rd }}$ vaccinations varied among breeds as measured by HI titer as follows. In Arsi chicken, the log HI titer range was: $1^{\text {st }} 2-4,2^{\text {nd }} 5-8,3^{\text {rd }} 5-7$; Horro $1^{\text {st }} 1-4,2^{\text {nd }} 2-8,3^{\text {rd }} 4-9$; Cheffe $1^{\text {st }}$ $4-8,2^{\text {nd }} 4-11,3^{\text {rd }} 3-12$; Fayoumi $1^{\text {st }} 3-9,2^{\text {nd }} 4-11,3^{\text {rd }} 7-12$; Tepi $1^{\text {st }} 1,4,6-8,2^{\text {nd }}$ $1-8,3^{\text {rd }} 4-9$; and Bovans $1^{\text {st }} 1-3,2^{\text {nd }} 1,2,4-8,3^{\text {rd }} 5-12$. Some chickens in the Tepi ecotype had low HI titer from the rest of the group after a single vaccination suggesting within ecotype variation. Second and $3^{\text {rd }}$ round LaSota vaccination increased HI titer in all the chicken ecotypes except some in Tepi after $2^{\text {nd }}$ vaccination (Table 1).

These proportions were used to compare the response of ecotype to ND vaccination. The HI log titer value of 2 was measured in 50\% of Arsi, 6 in $44 \%$ of Cheffe, 2 and 4 in 33\% (each) of Horro, 6 in 40\% of Tepi, 7 in 35\% of Jarso, 6 in 33\% of Fayoumi and 2 in $60 \%$ of Bovans following the $1^{\text {st }}$ vaccination (Table 1). In general, variation between and within each chicken ecotype in the HI response was consistently observed using proportions (\%) of chickens with specific HI 
titer following $1^{\text {st }}, 2^{\text {nd, }}$ and $3^{\text {rd }}$ vaccination (Table 1 ). In this study, Bovans had a lower HI titer than some Ethiopian local chicken ecotypes and Fayoumi.

Table 1. Proportion (\%) of individual chickens within seven chicken ecotypes with specific HI titer $\left(\log _{2}\right)$ and ranges of the HI titers during $1^{\text {st }}, 2^{\text {nd, }}$ and $3^{\text {rd }}$ round ND vaccination.

\begin{tabular}{|c|c|c|c|c|c|c|c|c|c|c|c|c|c|}
\hline \multirow[t]{2}{*}{ Breed } & \multicolumn{3}{|c|}{ Vaccination } & \multicolumn{10}{|c|}{ HI titer $\left(\log _{2}\right)$ ranges from 1 to 12} \\
\hline & & 1 & 2 & 3 & 4 & 5 & 6 & 7 & 8 & 9 & 10 & 11 & 12 \\
\hline \multirow[t]{3}{*}{ Arsi } & $1^{\text {st }}$ & - & 50 & 25 & 25 & - & - & - & - & - & - & - & - \\
\hline & $2^{\text {nd }}$ & - & - & - & - & 50 & - & 25 & 25 & - & - & - & - \\
\hline & $3^{\text {rd }}$ & - & - & - & - & 13 & 13 & 38 & - & 13 & - & 25 & - \\
\hline \multirow[t]{3}{*}{ Bovans } & $1^{\text {st }}$ & 30 & 60 & 10 & - & - & - & - & - & - & - & - & - \\
\hline & $2^{\text {nd }}$ & 10 & 20 & - & 20 & 20 & 10 & 10 & 10 & - & - & - & - \\
\hline & $3^{\text {rd }}$ & - & - & - & - & 16 & 5 & 16 & - & 21 & 11 & 5 & 26 \\
\hline \multirow[t]{3}{*}{ Cheffe } & $1^{\text {st }}$ & - & - & - & 8 & 8 & 44 & 31 & 8 & - & - & - & - \\
\hline & $2^{\text {nd }}$ & - & - & - & 3 & 14 & 26 & 23 & 11 & 6 & 11 & 6 & - \\
\hline & $3^{\text {rd }}$ & - & - & 1 & 3 & 15 & 12 & 15 & 10 & 10 & 4 & 7 & 22 \\
\hline \multirow[t]{3}{*}{ Fayoumi } & $1^{\text {st }}$ & - & - & 6 & 8 & 19 & 33 & 8 & 19 & 6 & - & - & - \\
\hline & $2^{\text {nd }}$ & - & - & - & 3 & 19 & 17 & 25 & 28 & 3 & - & 6 & - \\
\hline & $3^{\text {rd }}$ & - & - & - & - & - & - & 12 & 20 & 17 & 13 & 10 & 28 \\
\hline \multirow[t]{3}{*}{ Horro } & $1^{\text {st }}$ & 22 & 33 & 11 & 33 & - & - & - & - & - & - & - & - \\
\hline & $2^{\text {nd }}$ & - & 11 & 22 & - & 56 & 11 & - & - & - & - & - & - \\
\hline & $3^{\text {rd }}$ & - & - & - & 5 & 25 & 20 & 35 & 10 & 5 & - & - & - \\
\hline \multirow[t]{3}{*}{ Jarso } & $1^{\text {st }}$ & - & - & 5 & 5 & 20 & 10 & 35 & 25 & - & - & - & - \\
\hline & $2^{\text {nd }}$ & - & - & - & 10 & 5 & 10 & 10 & 30 & 15 & 20 & - & - \\
\hline & $3^{\text {rd }}$ & - & - & - & - & - & - & 14 & 5 & 30 & 8 & 16 & 27 \\
\hline \multirow[t]{3}{*}{ Tepi } & $1^{\text {st }}$ & 7 & - & - & 13 & - & 40 & 33 & 7 & - & - & - & - \\
\hline & $2^{\text {nd }}$ & 7 & 21 & 7 & 14 & 21 & 14 & 7 & 7 & - & - & - & - \\
\hline & $3^{\text {rd }}$ & - & - & - & 14 & 7 & 18 & 25 & 32 & 4 & - & - & - \\
\hline
\end{tabular}

\section{Ecotype differences to ND vaccination (unimodal or bimodal HI re- sponses)}

The immune response of each chicken ecotype/breed towards HI titer varied. The frequency of HI titer was different among breeds despite receiving the same vaccination. After the first $\mathrm{HB} 1$ vaccination on day 21, the serological response of Bovans had a mono-modal HI titer and peaked at HI titer 2 in $60 \%$ of 
the chickens. Chaffe also tends to have a uni-modal HI titer of 6 . The remaining chicken ecotypes had a bi-modal HI titer; however, peaks fall at different HI titer levels. Tepi ecotype had three peaks. This indicated the presence of subpopulations (diversities) within each ecotype, in addition to ecotype differences. Bovans and Cheffe seem to have less diversity (homogeneity) (Fig. 2).

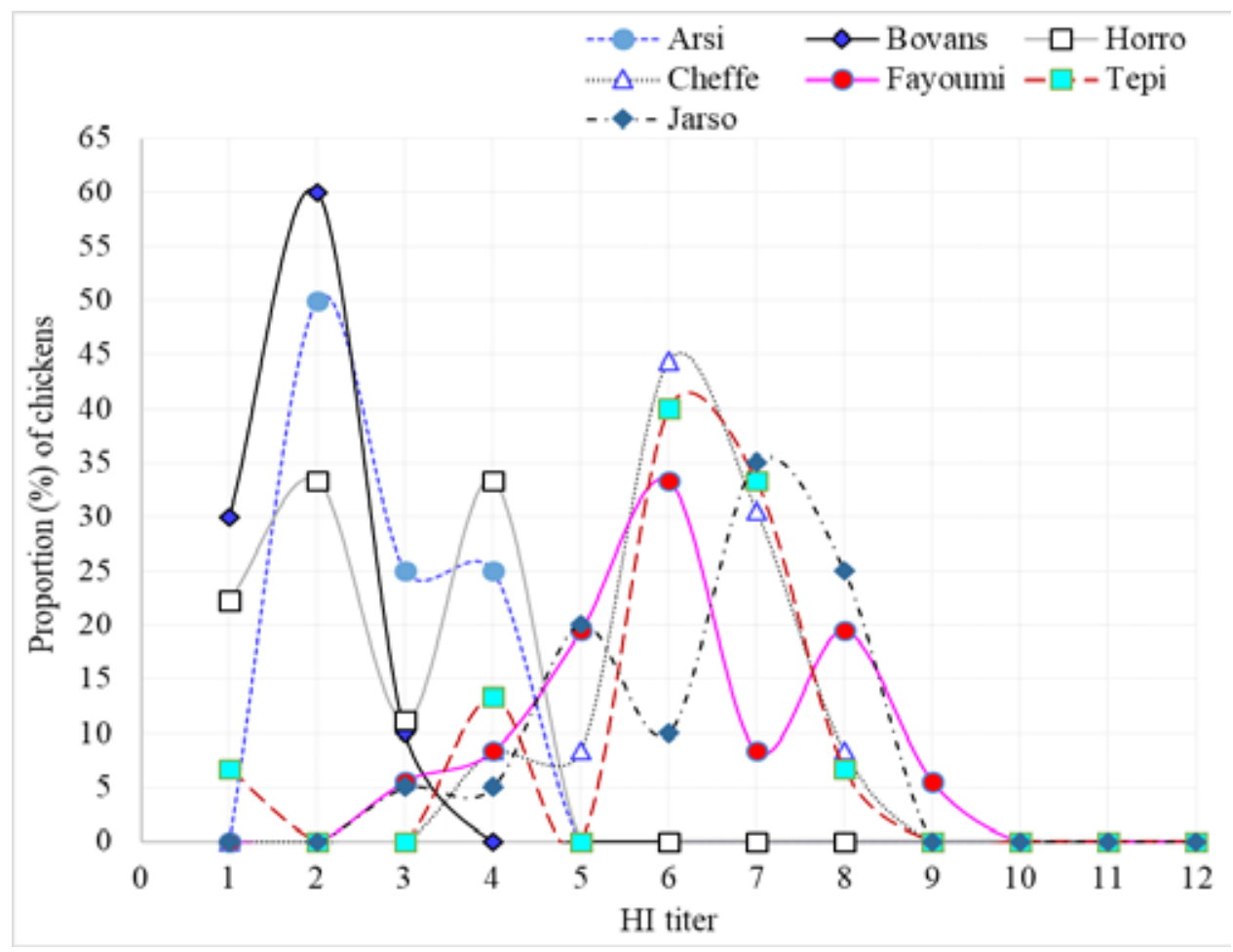

Figure 2. Differences in proportion (\%) of chickens displaying specific HI titer after a single ND vaccination among seven chicken ecotypes. The uni- or bimodal HI responses within each ecotype revealed the presence of diversities (subpopulations) in each chicken ecotype.

Similarly, following booster vaccination (i.e., $2^{\text {nd }}$ round vaccination), the curve of HI titer of the different ecotypes showed unique patterns for each ecotype whereby two or more models were showing the presence of subpopulations within each ecotype with a varying HI response to the ND vaccination (Fig 3). 


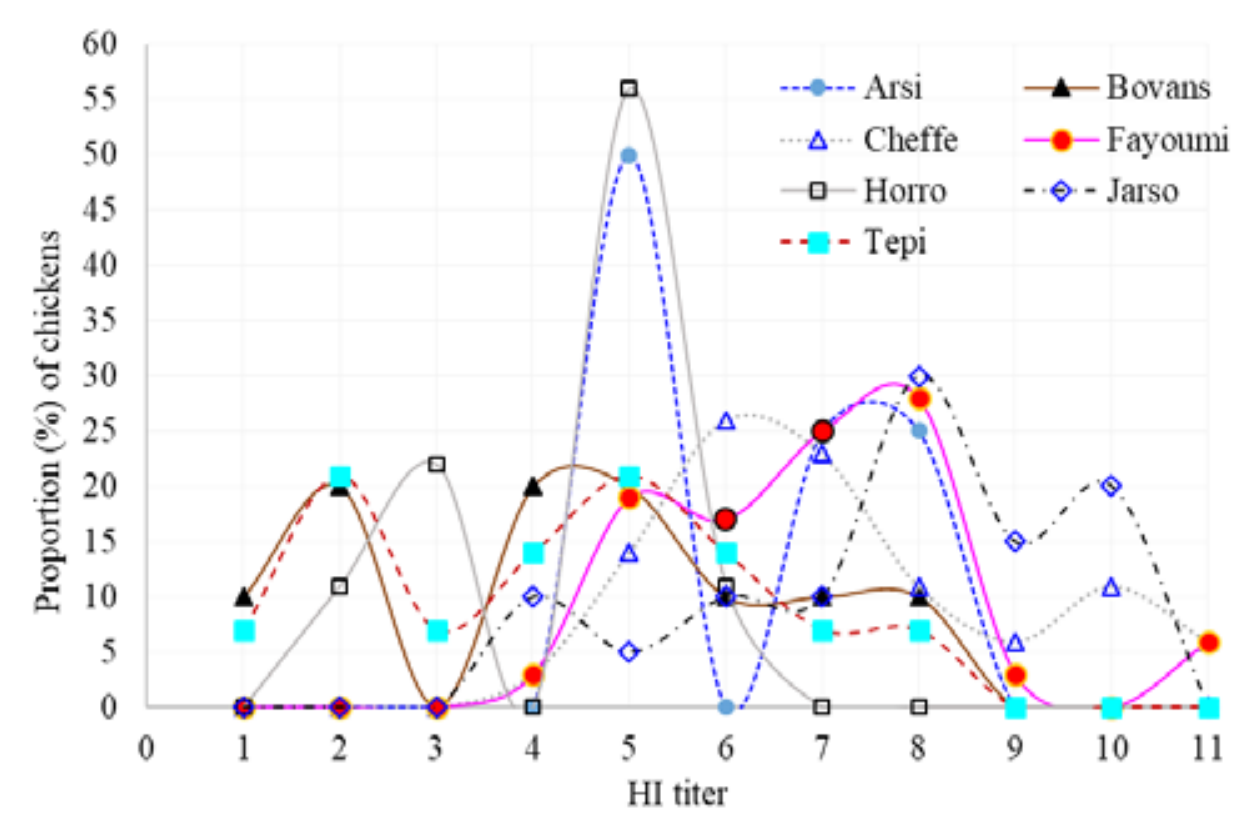

Figure 3. Differences in proportion (\%) of chickens displaying specific HI titer after two-round ND vaccinations among seven chicken ecotypes.

\section{HI antibody titer response of the chicken types to NDV vaccine strain}

There was a significant difference in HI titer across the sequential booster dosing of ND vaccination where the HI rises with increasing intensity of booster dosing. In this regard, post-vaccination geometric mean HI titers were significantly $(P<0.001)$ higher within each ecotype at 3 -times vaccination $>2$-times vaccination $>1$-time vaccination (Fig. 4$)$. A significant $(P<0.001)$ difference was noticed in mean log HI titer at 35, 65, and 135 days among chicken ecotypes. At day 35 the mean log HI titer ranked as Jarso $>$ Tepi $>$ Cheffe $>$ Fayoumi $>$ Arsi $>$ Horro $>$ Bovans. Similarly, at day 65 the mean HI titer rank as Jarso $>$ Cheffe $>$ Fayoumi $>$ Arsi $>$ Bovans $>$ Tepi $>$ Horro (Fig. 4). The mean $\log$ HI titer for Arsi, Horro, and Bovans was less $\left(\log _{2}<3\right)$ at day 35 following the first vaccination. The lowest titer was observed in Bovans (1.80), while the highest was observed for Jarso (6.51). Fayoumi, Chefe, Tepi, and Jarso had mean log HI titers above 5. Following booster dose on day 50 of age, serum sampling on day 65 of age showed that Horro ecotypes (3.90) had still lower HI titer followed by Tepi (3.92) and Bovans (4.40). On day 65, Jarso chickens maintained high titer (7.80) followed by Chefe (7.18) and Fayoumi (6.99). The 
HI titer in the Arsi ecotype increased (6.25), but the antibody level declined in Tepi at day 65 . The third-round vaccination increased the mean HI titer of all chicken breeds above $\log _{2}{ }^{2}>5$.

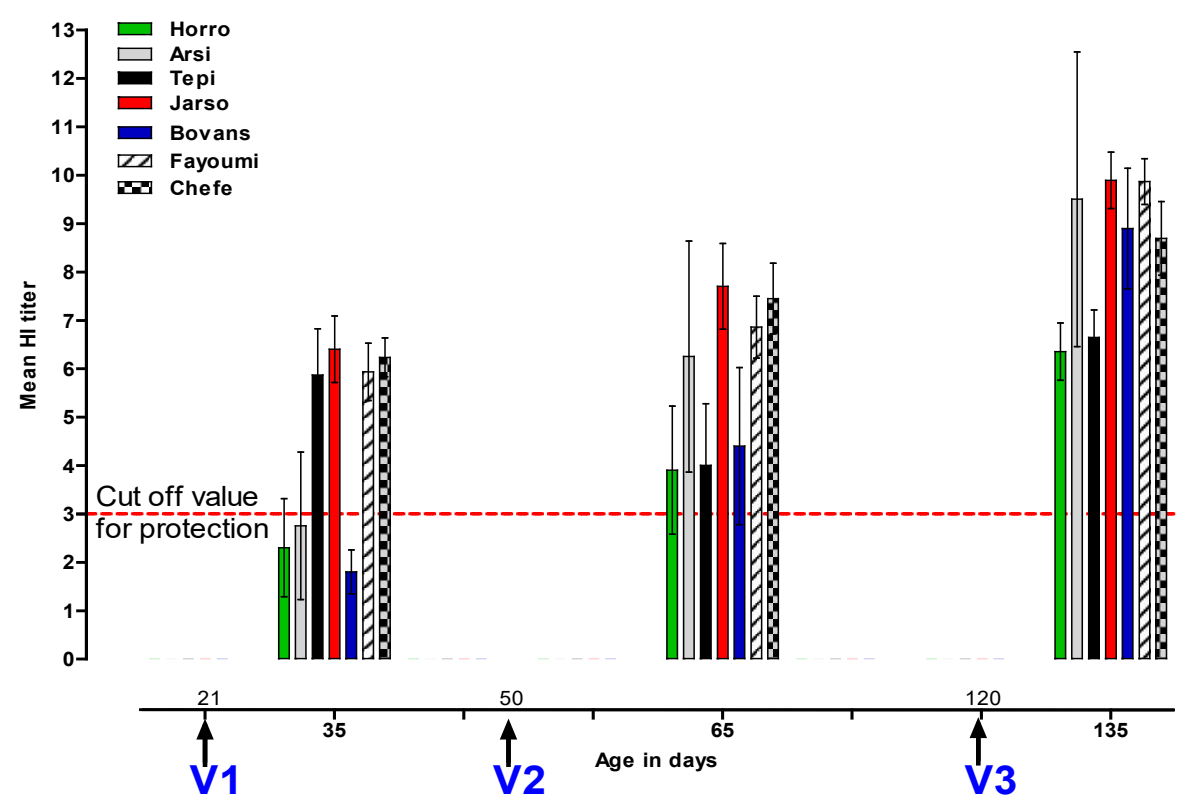

Figure 4. The HI titer of chicken ecotypes during three times ND immunization (V1 first vaccination, V2 second vaccination, V3 third vaccination).

\section{Proportion of chickens with protective titer $(\log 2 \geq 3)$ after vaccina- tion}

All (100\%) of Cheffe, Jarso, and Faoumi chickens acquired a protective HI titer after a single vaccination. However, $10 \%, 40 \%, 50 \%$, and $93 \%$ of Bovans, Horro, Arsi, and Tepi, respectively, had HI titer above the cut-off value after a single vaccination (Fig 5). Administering the second vaccination significantly increased the proportion of chickens with HI titer above the protective cut-off value in Bovans to $70 \%$, Horro to $80 \%$, and Arsi to $100 \%$. Conversely, in the Tepi ecotype, HI titer decreased below protective level after the second vaccination, even lower than the $1^{\text {st }}$ first immunization. The third vaccinationinduced HI titer was above the protective level in all ecotypes. 


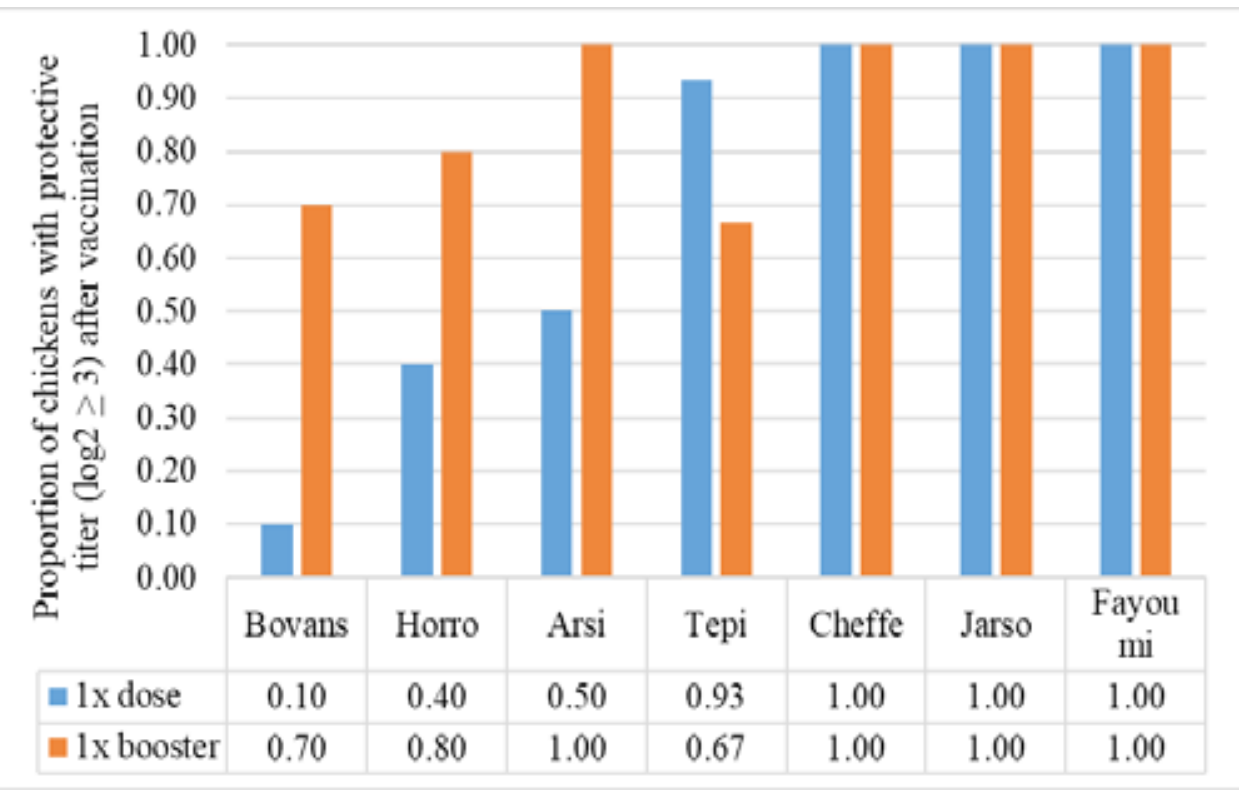

Figure 5. The proportion of chickens with protective HI titer $(\log 2 \geq 3)$ after first (blue color) and second (yellow color) vaccinations. Key: 1x (blue color) indicates one-time first vaccination to naïve chickens. 1x (yellow color) indicates one-time booster vaccination.

\section{Discussion}

Seasonal ND outbreaks are common in multiple geographical locations in Ethiopia (Mulisa et al., 2014). This suggests the presence of velogenic strains in the local viral pool (Diel et al., 2012; Snoeck et al., 2013; Fentie et al., 2014b; Mulisa et al., 2014; Bari et al., 2020), or periodic evolution of a novel velogenic strain, or spillover from wild birds (Alexander, 2011; Grubaugh et al., 2015). Ethiopia has never launched vaccination in the village backyard production system (Terfa et al., 2018) although ND vaccine is available in the country against the disease (Nasser et al., 2000; Abdi et al., 2016). Limited field trial on ND vaccine efficacy under field condition has indicated that HB1 and La Sota vaccination induces only 50-60\% immunity (Degefa et al., 2004) showing outbreaks and marked pathological lesions (Ezema et al., 2009), and shedding of the virus in the vaccinated chicken population (Fentie et al., 2014a). This indicates that ND vaccines are providing suboptimum efficacy, probably due to vaccine virus-associated factors such as inadequate dose and frequency of vaccine prescription or vaccine mismatch. In the current study, however, we 
propose that host genetic differences can be one of the reasons for the low immune response in vaccinated chickens in the field.

Therefore, we compared the HI titer of seven chicken ecotypes to ND immunization at ages 21,50 , and 120 days followed by serum collection for immune response analysis at days 35,65 , and 135 . We used HI titer for measuring humoral (antibody) responses. Measuring HI antibody titer directly in sera is widely used for monitoring the induction of antibodies (Yeo et al., 2003). The HI titer of $\log _{2} \geq 3$ has been considered the standard cut-off value for deciding effective protective titer against velogenic ND viral challenge (van Boven et al., 2008; Abdi et al., 2016). Further study is required whether the same cutoff value in HI titer works for Ethiopian local chickens. A comparative study among Ethiopian ecotypes on the persistence of maternal immunity in chicks as well as different genotypes infection/challenge study is also required.

In this study, after a single vaccination, $100 \%$ of Cheffe, Jarso, and Fayoumi chickens acquired HI titer above the protective level $\left(>\log _{2}{ }^{3}\right)$; however, $10 \%$, $40 \%$, 50\%, and 93\% of Bovans, Horro, Arsi, and Tepi, respectively, had HI titer $>$ greater than $\log _{2}{ }^{3}$. We have reported that the mean HI titer $\log _{2}{ }^{(3-5)}$ and $\log _{2}{ }^{(2-3)}$ corresponds to $70-80$ and $35-60 \%$ survival rates in infected chickens (Abdi et al., 2016). Chickens of different genetic backgrounds have differences in immune response (antibody titer) following vaccination (Newport et al., 2004; Liu et al., 2009). Geographic location, the season of birth, and the timing of vaccination can make the immune response of the host strong or weak during immunization (Hur et al., 2014). In the current study, environmental factors are controlled; the variation in the immune response was thus attributable to host genetics. It has been reported that each pathogen has a continuum of host population groups, ranging from highly susceptible to highly resistant (Hamilton, 1982; Lars et al., 2009; Grubaugh et al., 2015). This study revealed that the seven chicken ecotypes showed differences in the HI response to ND vaccine immunization, suggesting a single vaccination could not provide protective HI titers equally for some chickens of different genetic backgrounds.

After the second vaccination, the proportion of chickens with protective HI titer $\left(>\log _{2}{ }^{3}\right)$ increased: Bovans (70\%), Horro (80\%), and Arsi (100\%); but decreased from $93 \%$ (first vaccination) to $67 \%$ (second vaccination) in Tepi. The reason for the HI titer declines in the Tepi ecotype was unknown. On the other hand, HI titer in the remaining chicken breeds increased following booster vaccination. Differences in immune induction mechanisms and the persistence of 
antibodies post-vaccination have been observed among different breeds (Metcalf et al., 2015). ND antibodies are probably short-lived or incapable of mounting protection under real conditions since outbreaks are reported in vaccinated chickens in the field (Kapczynski and King, 2005; Kapczynski et al., 2013). Another study has similarly indicated that maternal immunity in chicken is depleted within 10 days after hatching (Gharaibeh and Mahmoud, 2013) suggesting the passive immunity from mothers to their neonates (i.e. maternal immunity) is short-lived. However, we poorly know the longitudinal dimension of immune kinetics, dynamics, maintenance, and clearance (decay) of the immune memory and antibodies in different chicken breeds. A study reported that ND antibodies persist for some years in wild birds after a single vaccination (Ramos et al., 2014).

The goal of a successful vaccination program in the field is to control outbreaks through increasing herd immunity. Herd immunity in ND is achieved when greater than $85 \%$ of the flocks have HI titer $>\log _{2}{ }^{3}$, which is mostly achieved after two vaccinations (van Boven et al., 2008). In this study, two vaccinations were not enough to yield HI titer $>\log _{2}{ }^{3}$ among some breeds such as Bovans, Horro, and Tepi compared to the remaining chicken ecotypes. In this regard, after two vaccinations, Bovans, Horro, and Tepi achieved HI titer of only $70 \%$, $80 \%$, and $67 \%$, respectively; thus, required third vaccination to achieve the standard protective HI titer of more than $\log _{2}{ }^{3}$. Finally, $100 \%$ of chickens vaccinated three times achieved HI titer above the standard cut-off value of more than $\log _{2}{ }^{3}$ for protection in this study.

Overall, HI titer above $\log _{2}{ }^{5}$ was achieved in Bovans, Horro, and Tepi after the third vaccination; in Arsi ecotype after the second vaccination, and in Cheffe, Jarso, and Fayoumi after a single vaccination; the results of which indicating breed variation in the immune response to ND immunization. We did not investigate the outcome of the challenge group using velogenic ND strain in this study. We determined that the mean HI titer above $\log _{2}{ }^{5}$ corresponds to a $100 \%$ survival rate (Abdi et al., 2016), caution, though, is needed in grading the survival of chicken breeds using HI titer alone. In this regard, cellular immunity (Ahmed et al., 2007; Dalgaard et al., 2010) or both antibody and cellmediated immunity contribute to protection against ND in different breeds (Juul-Madsen et al., 2011; Kapczynski et al., 2013). Furthermore, one of the six innate immune genes is highly expressed in response to ND (Deist et al., 2017; Schilling et al., 2018). Protection in animals against disease involves a separate or usually combined interaction of innate and acquired immunity 
(humoral and cell-mediated). In this study, we did not address the role of interaction of the innate and acquired immunity in protecting chicken against Newcastle disease; hence further study is required.

In general, commercial Bovans breed had a lower HI titer than some Ethiopian local chicken ecotypes and Fayoumi in this study. The commercial White-leg horn breed had a lower HI titer than the local chicken breed (Liu et al., 2009). The same finding was reported between commercial Fayoumi and local breeds (Deist et al., 2017). This suggests that improved animals for high production are generally weak in producing a protective level of the immune response. Despite selection for specific traits, which increased productivity by $90 \%$ in commercial chickens, they lost genetic diversity by over $50 \%$ compared to their ancestors indicating an inverse relationship between both traits (Muir et al., 2008). Generally, one of the undesirable side effects of selection for high production efficiency in farm animals is a decline in their disease resistance and immune response to pathogens (Rauw et al., 1998).

Overall, the HI titer against ND was significantly higher in Jarso $>$ Cheffe $>$ Fayoumi $>$ Arsi $>$ Bovans $>$ Tepi $>$ Horro suggesting antibody titer and ND vaccine efficacy of the ND vaccine depends on host factors. Ethiopian chicken ecotypes have demonstrated varying levels of resistance to some diseases such as Marek's disease as well as have had varying levels of production performance (Duguma, 2009). In this regard, the susceptibility of Ethiopian chickens to natural Marek's disease infection under confined management has been ranked previously based on mortality from highest to lowest as follows: Jarso $>$ Tepi $>$ Horro $\geq$ Konso $\geq$ Tilili $>$ Fayoumi (Duguma et al., 2005; Duguma, 2009). They have been also ranked based on improved survival rate (i.e. vaccine protection) following vaccination against Marek's disease and showed improved survival rate in the rank order of Jarso > Horro > Fayoumi > Tepi (Duguma, 2006; Duguma, 2009).

\section{Conclusions}

Ethiopian chicken ecotypes have demonstrated varying levels of immune response to ND vaccination both within and between each ecotype suggesting the presence of diverse subtypes in a chicken population. The existence of chicken groups with higher, medium, and lower immune responses within each ecotype can be an indicator of the chickens' strategy for investing their genetic 
resources in immunity than production. Thus, our result suggests that the chicken ecotypes originated from the different geographical areas may carry a unique set of reservoir genes with special utility in their local ecological conditions, which probably affect vaccine effectiveness and herd immunity; hence invites further studies. We conclude that polymorphism in host genetics influences the mobilization of the host immune defense system for an operation against infection suggesting hosts with a weak immune response may be a good hiding refuge for ND. Thus, a blanket ND vaccination program based on results derived from a single breed might not be realistic to infer for all breeds thriving in different geographical areas. In this regard, a robust comparative evaluation of the innate and acquired immunity responses is essential among chicken ecotypes during comparative experimental infection, immunity, and vaccine trial studies.

\section{Ethical approval}

Addis Ababa University College of Veterinary Medicine and Agriculture (Ref. 221/3/4257/2009) provided ethical approval for the experimental trial.

\section{Acknowledgments}

Ethiopian Agricultural Research Institute to the National Agricultural Research Fund for Rural Capacity Building Project funded this work (Ref. 221/3/4257). Addis Ababa University College of Veterinary Medicine and Agriculture hosted the implementation of the study activities within its premises. NVI did the HI titer.

\section{References}

Abdi, R.D., Amsalu, K., Merera, O, et al. 2016. Serological response and protection level evaluation in chickens exposed to grains coated with I2 Newcastle disease virus for effective oral vaccination of village chickens. BMC Vet. Res., 12, 150. doi: 10.1186/ s12917-016-0785-6.

Afonso, C.L., 2021. Virulence during Newcastle disease viruses cross-species adaptation. Viruses, 13(1),110.

Ahmed, K.A., Saxena, V.K., Ara. A., et al., 2007. Immune response to Newcastle disease virus in chicken lines divergently selected for cutaneous hypersensitivity. Int. J. Immunogenet., 34, 445-455. doi: 10.1111/j.1744-313X.2007.00722. x. 
Alexander, D.J., 2011. Newcastle disease in the European Union 2000 to 2009. Avian Pathol., 40, 547-558. doi: 10.1080/03079457.2011.618823.

Ashenafi, H., Tadesse, S., Medhin, G. and Tibbo, M., 2004. Study on coccidiosis of scavenging indigenous chickens in central Ethiopia. Trop. Anim. Health Prod., 36, 693-701. doi: 10.1023/B: TROP.0000042852.82477.af.

Bari, F.D., Gelaye, E., Tekola, B.G., Harder, T., Beer, M. and Grund, C., 2020. Antigenic and molecular characterization of virulent Newcastle disease viruses circulating in Ethiopia between 1976 and 2008. Vet. Med.: Res. Reports, 12, 129.

Bettridge J.M., Lynch, S.E., Brena, M.C., et al., 2014. Infection-interactions in Ethiopian village chickens. Prev. Vet. Med., 117, 358-366. doi: 10.1016/j.prevetmed.2014.07.002.

Chaka, H., Goutard, F., Bisschop, S.P.R. and Thompson, P.N., 2012. Seroprevalence of Newcastle disease and other infectious diseases in backyard chickens at markets in Eastern Shewa zone, Ethiopia. Poult. Sci., 91, 862-869. doi: 10.3382/ps.201101906.

Chaka, H., Goutard, F., Gil, P., et al., 2013a. Serological and molecular investigation of Newcastle disease in household chicken flocks and associated markets in Eastern Shewa zone, Ethiopia. Trop. Anim. Health Prod., 45,705-714. doi: 10.1007/s11250012-0278-y.

Chaka, H., Goutard, F., Roger, F., et al., 2013b. Household-level risk factors for Newcastle disease seropositivity and incidence of Newcastle disease virus exposure in backyard chicken flocks in Eastern Shewa zone, Ethiopia. Prev. Vet. Med., 109, 312-320. doi: 10.1016/j.prevetmed.2012.10.003.

Dalgaard, T.S., Norup, L.R., Pedersen A.R., et al., 2010. Flow cytometric assessment of chicken T cell-mediated immune responses after Newcastle disease virus vaccination and challenge. Vaccine, 28, 4506-4514. doi: 10.1016/j.vaccine.2010.04.044.

Dana, N. 2011. Breeding programs for indigenous chicken in Ethiopia Analysis of diversity in production systems and chicken populations. Ph.D. Thesis, Wageningen University.

Dana, N., Dessie, T., van der Waaij, L.H. and van Arendonk, J.A.M. 2010a. Morphological features of indigenous chicken populations of Ethiopia. Anim. Genet., 46, 11-23. doi: 10.1017/S2078633610000652.

Dana, N., van der Waaij, L.H., Dessie, T. and van Arendonk, J.A.M., 2010b. Production objectives and trait preferences of village poultry producers of Ethiopia: implications for designing breeding schemes utilizing indigenous chicken genetic resources. Trop. Anim. Health Prod., 42,1519-1529. doi: 10.1007/s11250-010-9602-6. 
de Almeida, R.S., Hammoumi, S., Gil, P., et al., 2013. New avian paramyxoviruses type I strains identified in Africa provide new outcomes for phylogeny reconstruction and genotype classification. PLOS ONE, 8, e76413. doi: 10.1371/journal.pone.0076413

Degefa, T., Dadi, L., Yami, A., et al. 2004. Technical and economic evaluation of different methods of Newcastle disease vaccine administration. J. Vet Med. A, 51, 365-369. doi: 10.1111/j.1439-0442.2004.00658.x.

Deist, M.S., Gallardo, R.A., Bunn, D.A., et al., 2017. Novel mechanisms revealed in the trachea transcriptome of resistant and susceptible chicken lines following infection with Newcastle disease virus. Clin. Vaccine. Immunol., 24, e00027-17. doi: 10.1128/CVI.00027-17.

Dessie, T. 2003. Phenotypic and genetic characterization of local chicken ecotypes in Ethiopia. Ph.D. Thesis, Humboldt University of Berlin.

Diel, D.G., da Silva, L.H.A., Liu, H., et al., 2012. Genetic diversity of avian paramyxovirus type 1: Proposal for a unified nomenclature and classification system of Newcastle disease virus genotypes. Infect. Genet. Evol., 12, 1770-1779. doi: 10.1016/j. meegid.2012.07.012

Dimitrov, K.M., Ramey, A.M., Qiu, X., Bahl, J., and Afonso, C.L., 2016. Temporal, geographic, and host distribution of avian paramyxovirus 1 (Newcastle disease virus). Infect. Genet. Evol., 39, 22-34.

Dortmans, J.C.F.M., Peeters, B.P.H., and Koch G., 2012. Newcastle disease virus outbreaks: Vaccine mismatch or inadequate application? Vet. Microbiol., 160, 17-22. doi: 10.1016/j.vetmic.2012.05.003.

Duguma, R., 2009. Understanding the role of indigenous chicken during the long walk to food security in Ethiopia. LRRD, 21: http://lrrd.org/lrrd21/8/dugu2116.htm.

Duguma, R., 2006. Phenotypic characterization of some indigenous chicken ecotypes of Ethiopia. LRRD, 18, 13. 1http://www.cipav.org.co/lrrd//rrd18/9/dugu18131.htm.

Duguma, R., Dana, N., and Yami, A. 2006. Marek's disease vaccination opened the door to rear indigenous chickens of Ethiopia under confined management. Int. J. Appl. Res. Vet. Med., 4, 121.

Duguma, R., Yami, A., Dana, N., et al., 2005. Marek's disease in local chicken strains of Ethiopia reared under confined management regime in central Ethiopia. Revue Med. Vet., 156, 541-546.

Ezema, W.S., Okoye, J.O.A., and Nwanta, J.A., 2009. LaSota vaccination may not protect against the lesions of velogenic Newcastle disease in chickens. Trop. Anim. Health. Prod., 41, 477-484. doi: 10.1007/s11250-008-9210-x. 
Fentie, T., Dadi, K., Kassa, T., et al., 2014a. Effect of vaccination on transmission characteristics of highly virulent Newcastle disease virus in experimentally infected chickens. Avian Pathol., 43, 420-426. doi: 10.1080/03079457.2014.951832.

Fentie, T., Heidari, A., Aiello, R., et al., 2014b. Molecular characterization of Newcastle disease viruses isolated from rural chicken in northwest Ethiopia reveals the circulation of three distinct genotypes in the country. Trop. Anim. Health Prod., 46, 299-304. doi: 10.1007/s11250-013-0487-z.

Gari, A.T., Getnet, M., and Nigatu, L. 2018. Observed and future climate variability, trend, and extremes in central highlands of Ethiopia: A case study at Arsi Robe, Asasa, Debre Zeit and Kulumsa Areas. Environ. Pollut. Climate. Change., 2(163), 10-4172.

Gharaibeh, S., and Mahmoud, K., 2013. Decay of maternal antibodies in broiler chickens. Poult. Sci., 92, 2333-2336. doi: 10.3382/ps.2013-03249.

Goraga, Z., Weigend, S., and Brockmann, G. 2012. Genetic diversity and population structure of five Ethiopian chicken ecotypes. Anim. Genet., 43, 454-457. doi: 10.1111/j.1365-2052.2011.02270.x.

Grubaugh, N.D., Smith, D.R., Brackney, D.E., et al., 2015. Experimental Evolution of an RNA Virus in Wild Birds: Evidence for host-dependent impacts on population structure and competitive fitness. PLOS Pathog., 11, e1004874. doi: 10.1371/journal.ppat. 1004874 .

Gulilat, L., Tegegne, F., and Demeke, S., 2021. Hatchery and broody technologies and least-cost ration practice for poultry production improvement in Ethiopia. Cogent. Food. Agric., 7(1), 1913793.

Hamilton, W.D., 1982. Pathogens as causes of genetic diversity in their host populations. In: Anderson RM, May RM (eds) Population Biology of Infectious Diseases. Springer Berlin Heidelberg, pp 269-296.

Hassen, H., Neser, F.W.C., De Kock, A., and van Marle-Köster, E., 2009. Study on the genetic diversity of native chickens in northwest Ethiopia using microsatellite markers. Afr. J. Biotechnol., 8 (7), 1347-1353.

Hassen, H.H., 2007. Phenotypic and genetic characterization of indigenous chicken populations in Northwest Ethiopia. Ph.D. Thesis, University of the Free State.

Hur, Y-G., Gorak-Stolinska, P., Lalor, M.K., et al., 2014. Factors affecting the immunogenicity of BCG in infants, a study in Malawi, The Gambia, and the UK. BMC Infect. Dis., 14, 184. doi: 10.1186/1471-2334-14-184.

Juul-Madsen, H.R., Norup, L.R., Jørgensen, P.H., et al., 2011. Crosstalk between innate and adaptive immune responses to infectious bronchitis virus after vaccination 
and challenge of chickens varying in serum mannose-binding lectin concentrations. Vaccine, 29, 9499-9507. doi: 10.1016/j.vaccine.2011.10.016.

Kapczynski, D.R., Afonso, C.L., and Miller, P.J., 2013. Immune responses of poultry to Newcastle disease virus. Dev. Comp. Immunol., 41, 447-453. doi: 10.1016/j. dci.2013.04.012.

Kapczynski, D.R., and King, D.J., 2005. Protection of chickens against overt clinical disease and determination of viral shedding following vaccination with commercially available Newcastle disease virus vaccines upon challenge with highly virulent virus from the California 2002 exotic Newcastle disease outbreak. Vaccine, 23, 3424-3433. doi: 10.1016/j.vaccine.2005.01.140.

Koenen, M.E., Boonstra-Blom, A.G. and Jeurissen, S.H., 2002. Immunological differences between layer- and broiler-type chickens. Vet. Immunol. Immunopathol., 89, 47-56. doi: 10.1016/S0165-2427(02)00169-1

Liu, L.B., Wu, C.M., Wen, J., et al., 2009. Association of SNPs in exon 2 of the MHC B-F gene with immune traits in two distinct chicken populations: Chinese Beijing-You and White Leghorn. Acta Agric. Scand.- A: Anim. Sci., 59, 4-11. doi: 10.1080/09064700902988905.

Luu, L., Bettridge, J., Christley, R.M., et al., 2013. Prevalence and molecular characterization of Eimeria species in Ethiopian village chickens. BMC Vet. Res., 9, 208. doi: 10.1186/1746-6148-9-208.

Melesse, A., and Negesse, T., 2011. Phenotypic and morphological characterization of indigenous chicken populations in the southern region of Ethiopia. Anim. Genet., 49, 19-31. doi: 10.1017/S2078633611000099.

Metcalf, C.J.E., Andreasen, V., Bjørnstad, O.N., et al., 2015. Seven challenges in modeling vaccine-preventable diseases. Epidemics, 10, 11-15. doi: 10.1016/j.epi$\operatorname{dem} .2014 .08 .004$.

Miguel, E., Grosbois, V., Berthouly-Salazar, C., et al., 2013. A meta-analysis of observational epidemiological studies of Newcastle disease in African agro-systems, 1980-2009. Epidemiol. Infect., 141, 1117-1133. doi: 10.1017/S0950268812002610.

Muir, W.M., Wong, G.K-S., Zhang, Y., et al., 2008. Genome-wide assessment of worldwide chicken SNP genetic diversity indicates a significant absence of rare alleles in commercial breeds. Proc. Natl. Acad. Sci. USA., 105, 17312. doi: 10.1073/ pnas.0806569105.

Mulisa, D.D., W/Kiros, M.K., Alemu, R.B., et al., 2014. Characterization of Newcastle disease virus and poultry-handling practices in live poultry markets, Ethiopia. SpringerPlus, 3, 459. doi: 10.1186/2193-1801-3-459. 
Nasser, M., Lohr, J.E., Mebratu, G.Y., et al., 2000. Oral Newcastle disease vaccination trials in Ethiopia. Avian Pathol., 29, 27-34. doi: 10.1080/03079450094243.

Newport, M.J., Goetghebuer, T., Weiss, H.A., et al., 2004. Genetic regulation of immune responses to vaccines in early life. Genes Immun., 5, 122.

Ostfeld, R.S., and Keesing, F., 2012. Effects of host diversity on infectious disease. Annu. Rev. Ecol. Evol. Syst., 43, 157-182. doi: 10.1146/annurev-ecolsys-102710-145022.

Lars, R., Andrea, G. L, and Andrew, R.F., 2009. Decomposing health: tolerance and resistance to parasites in animals. Philos. Trans. R. Soc. B: Biol. Sci., 364, 37-49. doi: 10.1098/rstb.2008.0184.

Ramos, R., Garnier, R., González-Solís, J. and Boulinier, T., 2014. Long antibody persistence and transgenerational transfer of immunity in a long-lived vertebrate. $\mathrm{Am}$. Nat., 184, 764-776. doi: 10.1086/678400.

Rauw, W.M., Kanis, E., Noordhuizen-Stassen, E.N., and Grommers, F.J., 1998. Undesirable side effects of selection for high production efficiency in farm animals: a review. Livest. Prod. Sci., 56(1), 15-33.

Sarba, E.J., Bayu, M.D., Gebremedhin E.Z., et al., 2019. Gastrointestinal helminths of backyard chickens in selected areas of West Shoa Zone Central, Ethiopia. Vet. Parasitol. Reg. Stud. Reports., 15, 100265. doi: 10.1016/j.vprsr.2019.100265.

Schilling, M.A., Katani, R., Memari, S., et al., 2018. Transcriptional innate immune response of the developing chicken embryo to Newcastle disease virus infection. Front. Genet., 9, 61. doi: 10.3389/fgene.2018.00061.

Snoeck, C.J., Owoade, A.A., Couacy-Hymann, E., et al., 2013. High genetic diversity of Newcastle disease virus in poultry in West and Central Africa: Cocirculation of genotype XIV and newly defined genotypes XVII and XVIII. J. Clin. Microbiol., 51, 2250. doi: 10.1128/JCM.00684-13.

Terfa, Z.G., Garikipati, S., Kassie, G., et al., 2018. Eliciting preferences for attributes of Newcastle disease vaccination programmes for village poultry in Ethiopia. Prev. Vet. Med., 158, 146-151. doi: 10.1016/j.prevetmed.2018.08.004.

van Boven, M., Bouma, A., Fabri, T.H.F., et al., 2008. Herd immunity to Newcastle disease virus in poultry by vaccination. Avian Pathol., 37, 1-5. doi: 10.1080/03079450701772391.

Yeo, S., Nagy, E., and Krell, P.J., 2003. Indirect method for prediction of hemagglutination inhibition antibody titers to Newcastle disease virus in chickens by titration of antibodies in egg yolk. J. Vet. Diagn. Invest., 15, 184-187. doi: $10.1177 / 104063870301500216$. 